TRANSACTIONS OF THE

AMERICAN MATHEMATICAL SOCIETY

Volume 359, Number 4, April 2007, Pages 1499-1515

S 0002-9947(06)04153-5

Article electronically published on November 3, 2006

\title{
PRIMENESS, SEMIPRIMENESS AND LOCALISATION IN IWASAWA ALGEBRAS
}

\author{
KONSTANTIN ARDAKOV AND KENNETH A. BROWN
}

\begin{abstract}
Necessary and sufficient conditions are given for the completed group algebras of a compact $p$-adic analytic group with coefficient ring the $p$-adic integers or the field of $p$ elements to be prime, semiprime and a domain. Necessary and sufficient conditions are found for the localisation at semiprime ideals related to the augmentation ideals of closed normal subgroups. Some information is obtained about the Krull and global dimensions of the localisations. The results extend and complete work of A. Neumann and J. Coates et al.
\end{abstract}

\section{INTRODUCTION}

1.1. In recent years there has been increasing interest in noncommutative Iwasawa algebras. These are the completed group algebras

$$
\Lambda_{G}:=\quad \longleftarrow \lim \mathbb{Z}_{p}[G / U]
$$

where $\mathbb{Z}_{p}$ denotes the ring of $p$-adic integers, $G$ is a compact $p$-adic analytic group, and the inverse limit is taken over the open normal subgroups of $G$. Closely related is the epimorphic image $\Omega_{G}$ of $\Lambda_{G}$,

$$
\Omega_{G}:=\lim _{p} \mathbb{F}_{p}[G / U]
$$

where $\mathbb{F}_{p}$ is the field of $p$ elements. This increased interest has been driven by anticipated applications of these rings in number theory and arithmetic algebraic geometry; see for example [6] and the references there for more details.

1.2. These algebras were first defined and studied by Lazard in his celebrated paper [10]. There, he characterised compact $p$-adic analytic groups as precisely those topological groups containing an open normal uniform pro-p subgroup of finite index [7, Corollary 8.34]. Uniform pro- $p$ groups are defined at [7, Definition 4.1]. Lazard showed that when $G$ is uniform $\Lambda_{G}$ and $\Omega_{G}$ have the structure of noncommutative formal power series algebras. In particular, both $\Lambda_{G}$ and $\Omega_{G}$ are in this case local, meaning that the factor of each by its Jacobson radical is simple Artinian, [7. Exercise 6.2]. (In fact, $\Lambda_{G} / J\left(\Lambda_{G}\right) \cong \Omega_{G} / J\left(\Omega_{G}\right) \cong \mathbb{F}_{p}$.) Moreover (still assuming that $G$ is uniform), $\Lambda_{G}$ and $\Omega_{G}$ are filtered by descending chains of ideals, and the associated graded algebras are (commutative) polynomial algebras in finitely many

Received by the editors January 5, 2005.

2000 Mathematics Subject Classification. Primary 16P40, 16L30, 11R23, 20 C07.

Key words and phrases. Noncommutative Iwasawa theory, pro- $p$ group, completed group algebra, complete noetherian local ring, prime ring, semiprime ring, localisable ideal.

The first author thanks Christ's College, Cambridge, for financial support.

(C)2006 American Mathematical Society Reverts to public domain 28 years from publication 
variables over $\mathbb{F}_{p}$. It follows that $\Lambda_{G}$ and $\Omega_{G}$ are Noetherian domains with good homological properties. A very clear treatment of all this material can be found in [7.

If $G$ is now any compact $p$-adic analytic group, with normal uniform open subgroup $U$, say, then clearly

$$
\Lambda_{G} \cong \Lambda_{U} *(G / U),
$$

a crossed product of the local Noetherian domain $\Lambda_{U}$ by the finite group $G / U$. Thus $J\left(\Lambda_{U}\right) \subseteq J\left(\Lambda_{G}\right)$ by [14, Theorem 4.2], and hence $\Lambda_{G}$ is Noetherian and semilocal; that is, $\Lambda_{G} / J\left(\Lambda_{G}\right)$ is semisimple Artinian. Similar remarks apply to $\Omega_{G}$.

1.3. Primeness and semiprimeness. Notwithstanding the results summarised in (1.2), very little is known about the (2-sided) ideal structure of noncommutative Iwasawa algebras. The purpose of this paper is to take the initial steps towards remedying this. Recall that, if $G$ is any group,

$$
\Delta^{+}(G)=\left\{x \in G:\left|G: C_{G}(x)\right|<\infty \quad \text { and } \quad o(x)<\infty\right\} .
$$

This is a characteristic locally finite subgroup, generated by all the finite normal subgroups of $G$ [14, Lemma 5.1]. If $G$ is compact $p$-adic analytic, then $G$ has a torsion-free subgroup of finite index, since uniform groups are torsion-free 7 , Theorem 4.5], so that $\Delta^{+}(G)$ is finite, the unique maximal finite normal subgroup of $G$.

We begin with $\Omega_{G}$. The first two results are the analogues for Iwasawa algebras of well-known theorems for ordinary group algebras, proved by I. G. Connell and D. S. Passman in 1963 and 1962 [15, Theorems 4.2.10 and 4.2.13]. The proofs for Iwasawa algebras are however completely different from those for ordinary group algebras, relying crucially on the crossed product decomposition (11).

Theorem A. Let $G$ be a compact p-adic analytic group. Then $\Omega_{G}$ is prime if and only if $\Delta^{+}(G)=1$.

The definition and basic properties of the p-Sylow subgroups of a profinite group can be found at [21, $\S 2.2]$.

Theorem B. Let $G$ be a compact p-adic analytic group and let $P$ be a $p$-Sylow subgroup of $G$. The following are equivalent:

(i) $\Omega_{G}$ is semiprime;

(ii) $\Omega_{P}$ is semiprime;

(iii) $\Omega_{P}$ is prime;

(iv) $\Delta^{+}(P)=1$;

(v) $p \nmid\left|\Delta^{+}(G)\right|$.

As we show in Theorem $\mathrm{F}$, it is straightforward to deduce the analogue for $\Lambda_{G}$ of Theorem A. The analogue for $\Lambda_{G}$ of Theorem B, stating that $\Lambda_{G}$ is always semiprime for $G$ compact $p$-adic analytic, was noted in [13] - one simply appeals to the definition of $\Lambda_{G}$ as a projective limit of ordinary group rings of finite groups, over a characteristic zero coefficient ring, together with the fact that such group rings are semiprime. 
1.4. Zero divisors. We also characterise those $G$ for which $\Omega_{G}$ is a domain. The corresponding result for $\Lambda_{G}$ is due to Neumann [13].

Theorem C. Let $G$ be a compact p-adic analytic group. Then $\Omega_{G}$ is a domain if and only if $G$ is torsion-free.

1.5. Localisation at augmentation ideals. A semiprime ideal $I$ of the Noetherian $\operatorname{ring} R$ is said to be right localisable if the subset $\mathcal{C}_{R}(I):=\{c \in R$ : $c+I$ not a zero divisor in $R / I\}$ satisfies the right Ore condition. Left localisable ideals are defined analogously, and we say that $I$ is localisable if it is right and left localisable. When the localisation exists, it will be denoted $R_{I}$; this is a Noetherian semilocal ring whose Jacobson radical is $I R_{I}$. We say that the ideal $I$ satisfies the right Artin-Rees property if, for every right ideal $E$ of $R$, there exists $n \geqslant 1$ such that $E \cap I^{n} \subseteq E I$. The issue of whether a given ideal is localisable is intimately connected with the Artin-Rees property, so much so that, following [9], we shall say that the semiprime ideal $I$ is classically right localisable if it is right localisable and $I R_{I}$ satisfies the right Artin-Rees property in $R_{I}$; of course "right" will be omitted where appropriate. It may well be that the adjective "classical" is redundant here - in fact it is a long-standing question of Jacobson whether the Jacobson radical of a Noetherian ring always has the Artin-Rees property.

Let $G$ be a compact $p$-adic analytic group and $H$ a closed normal subgroup of $G$. Since any finite normal subgroup of $H$ is contained in $\Delta^{+}(H)$ and the product of two finite normal $p^{\prime}$-subgroups of $H$ is again a finite normal $p^{\prime}$-subgroup of $H$, we see that $H$ has a largest finite normal $p^{\prime}$-subgroup, which we will denote by $\Delta_{p^{\prime}}^{+}(H)$. This subgroup is clearly characteristic in $H$ and hence is normal in $G$.

Let $w_{H, G}$ denote the kernel of the natural map $\Omega_{G} \rightarrow \Omega_{G / H}$. From our main result on localisation (Theorem D in (3.1)), we can deduce

Theorem E. Let $G$ be a compact p-adic analytic group and let $H$ be a closed normal subgroup of $G$. Suppose that $\Omega_{G / H}$ is semiprime. Then the following are equivalent:

(i) $w_{H, G}$ is localisable;

(ii) $w_{H, G}$ is classically localisable;

(iii) $H / \Delta_{p^{\prime}}^{+}(H)$ is pro-p.

As before, there are versions of Theorems $\mathrm{D}$ and $\mathrm{E}$ for $\Lambda_{G}$ also; these are stated and proved in (4.3). Theorem $\mathrm{E}$ is the analogue for compact $p$-adic groups of results of J.E. Roseblade and P.F. Smith for ordinary group algebras of polycyclic-by-finite groups; see [15, Theorem 11.2.15].

In the case where $H$ is pro- $p$ and $G / H \cong \mathbb{Z}_{p}$, the localisability of the augmentation ideal $v_{H, G}=\operatorname{ker}\left(\Lambda_{G} \rightarrow \Omega_{G / H}\right)$ of $\Lambda_{G}$ is proved in [6, 2.4,2.6], a result which prompted the work described in this paragraph. Whereas the methods used in that paper are module-theoretic, our methods are more ring-theoretic in nature and are in fact natural extensions of the arguments used by Venjakob in [18, 5.4.1].

There, he proves Theorem $\mathrm{E}(\mathrm{iii}) \Rightarrow(\mathrm{i})$ and Theorem $\mathrm{H}(\mathrm{iii}) \Rightarrow(\mathrm{i})$ in the special cases when $H$ is uniform and $G=H \times \mathbb{Z}_{p}$ and when $H=\mathbb{Z}_{p}$ and $G=H \rtimes \mathbb{Z}_{p}[18$, Theorem 5.15], and conjectures that these results generalize to the case when $H$ is $p$-valued and $G / H$ is torsion-free in [18, Conjecture 5.14].

1.6. Properties of the localisations. In the last section of this paper, we consider the Krull dimension and homological properties of the localisations of $\Omega_{G}$ and 
$\Lambda_{G}$. Let $\Omega_{G, H}$ and $\Lambda_{G, H}$ denote the localisations of $\Omega_{G}$ and $\Lambda_{G}$ at $P_{H}$ and $I_{H}$, respectively. See (3.1) and (4.3) for the relevant definitions; we simply note here that if $H$ is a pro- $p$ group with $\Delta^{+}(G / H)=1$, then $P_{H}=w_{H, G}$ and $I_{H}=v_{H, G}$.

Theorem I. Let $G$ be a compact p-adic analytic group and let $H$ be a closed normal subgroup of $G$. Then

(i) $\mathcal{K}\left(\Omega_{H}\right) \leqslant \mathcal{K}\left(\Omega_{G, H}\right) \leqslant \operatorname{dim} H$,

(ii) $\mathcal{K}\left(\Lambda_{G, H}\right)=\mathcal{K}\left(\Omega_{G, H}\right)+1$.

It is proved in [1] that $\lambda(\mathfrak{h}) \leq \mathcal{K}\left(\Omega_{H}\right)$, where $\mathfrak{h}$ is the $\mathbb{Q}_{p}$-Lie algebra of $H$ and $\lambda(\mathfrak{h})$ is the maximum length $m$ of chains $0=\mathfrak{h}_{0}<\mathfrak{h}_{1}<\ldots<\mathfrak{h}_{m}=\mathfrak{h}$ of sub-Liealgebras of $\mathfrak{h}$. When $\mathfrak{h}$ is solvable, $\mathcal{K}\left(\Omega_{H}\right)=\operatorname{dim} H$ and so (i) consists of equalities, but in general $\mathcal{K}\left(\Omega_{H}\right)<\operatorname{dim} H$.

The parallel result on global dimensions is part of the following theorem. The unexplained terminology is defined in Section [5.4. When $H=G$ and $G$ has no elements of order $p$, we obtain an earlier result of Venjakob [19, Theorem 3.26].

Theorem J. Let $G$ be a compact p-adic analytic group and let $H$ be a closed normal subgroup of $G$. Then

(i) $\Omega_{G, H}$ is Auslander-Gorenstein of injective dimension $\operatorname{dim} H$.

(ii) $\Lambda_{G, H}$ is Auslander-Gorenstein of injective dimension $\operatorname{dim} H+1$.

(iii) If $G$ contains no element of order $p$, then $\Omega_{G, H}$ and $\Lambda_{G, H}$ have finite global dimension. In this case both algebras are Auslander-regular, and

$$
\operatorname{gld}\left(\Omega_{G, H}\right)=\operatorname{gld}\left(\Omega_{H}\right)=\operatorname{dim} H,
$$

while

$$
\operatorname{gld}\left(\Lambda_{G, H}\right)=\operatorname{gld}\left(\Lambda_{H}\right)=\operatorname{gld}\left(\Omega_{G, H}\right)+1 .
$$

(iv) If $\Omega_{G, H}$ or $\Lambda_{G, H}$ has finite global dimension, then the inverse image in $G$ of $\Delta^{+}(G / H)$ contains no elements of order $p$.

\section{Primeness And semiprimeness of $\Omega_{G}$}

2.1. Uniform pro- $-p$ groups. We begin with some technical results concerning $\Omega_{N}$ for uniform $N$. The following notation will be fixed for the rest of this paper:

$$
\epsilon= \begin{cases}2 & \text { if } p=2, \\ 1 & \text { otherwise. }\end{cases}
$$

Lemma. Let $N$ be a uniform pro-p group and let $\mathfrak{m}$ denote the maximal ideal of $\Omega_{N}$. Then

(i) $\left[\Omega_{N}, \mathfrak{m}^{k}\right] \leqslant \mathfrak{m}^{k+p^{\epsilon}-1}$ for all $k \geqslant 1$,

(ii) $\left(1+\mathfrak{m}^{p^{\epsilon}-1}\right) \cap N \leqslant N^{p^{\epsilon}}$.

Proof. To avoid confusion, we will denote the Lie commutator in $\Omega_{N}$ by $[a, b]:=$ $a b-b a$, and the group commutator in $N$ by $(x, y):=x^{-1} y^{-1} x y$.

Since $N$ is uniform and so in particular powerful, $(N, N) \leq N^{p^{\epsilon}}$ [7, Definition 3.1 and Lemma 3.4]. From this, it is easy to deduce that $\left[\Omega_{N}, \mathfrak{m}\right]=[\mathfrak{m}, \mathfrak{m}] \leqslant \mathfrak{m}^{p^{\epsilon}}$. Part (i) now follows by a straightforward induction.

Next, $g N^{p} \mapsto g-1+\mathfrak{m}^{2}$ gives an isomorphism of the groups $N / N^{p}$ and $\mathfrak{m} / \mathfrak{m}^{2}$, so $\left(1+\mathfrak{m}^{2}\right) \cap N=N^{p}$. If $p$ is odd, part (ii) follows, so assume that $p=2$. Choose a topological generating set $\left\{a_{1}, \ldots, a_{d}\right\}$ for $N$ and let $g \in\left(1+\mathfrak{m}^{3}\right) \cap N$. Since 
$\left(1+\mathfrak{m}^{3}\right) \cap N \leqslant N^{2}$ by the above, we can write $g=a_{1}^{2 \mu_{1}} \cdots a_{d}^{2 \mu_{d}}$ for some $\mu_{i} \in \mathbb{Z}_{2}$ by [7, Theorems 3.6(iii) and 3.7 and Proposition 1.28]. Set $b_{i}=a_{i}-1 \in \Omega_{N}$. Then

$$
g-1=\left(1+b_{1}^{2}\right)^{\mu_{1}} \cdots\left(1+b_{d}^{2}\right)^{\mu_{d}}-1 \equiv \bar{\mu}_{1} b_{1}^{2}+\ldots+\bar{\mu}_{d} b_{d}^{2} \quad \bmod \mathfrak{m}^{3},
$$

where $\bar{\mu}_{i}$ denotes the reduction of $\mu_{i}$ modulo 2. By [7, Theorem 7.24], the elements $b_{i}^{2}$ are linearly independent modulo $\mathfrak{m}^{3}$, so $\mu_{i} \in 2 \mathbb{Z}_{2}$ for all $i$. Hence $g \in N^{4}$ as required.

Recall that if $N$ is uniform, then $\Omega_{N}$ is a Noetherian domain [7, Corollary 7.25], and as such has a division ring of quotients $D_{N}$ [12, Theorem 2.1.14]. Define the degree $\operatorname{deg} r$ of a nonzero element $r \in \Omega_{N}$ to be the greatest nonnegative integer $k$ such that $r$ is in the $k$ th power of the maximal ideal of $\Omega_{N}$. The following result will be crucial to our proof of Theorem A.

Proposition. Let $N$ be a uniform pro-p group and let $\varphi \in \operatorname{Aut}(N)$. Suppose that the natural extension of $\varphi$ to $D_{N}$ is inner. Then $[N, \varphi] \subseteq N^{p^{\epsilon}}$.

Proof. As in Lemma 2.1, let $\mathfrak{m}$ be the maximal ideal of $\Omega_{N}$. By assumption, we can find a nonzero element $x \in D_{N}$ such that

$$
x^{-1} g x=g^{\varphi} \quad \text { for all } g \in N .
$$

We can also find nonzero elements $a, b, c, d \in \Omega_{N}$ such that $x=b d^{-1}=a^{-1} c$; note that this forces

$$
a b=c d .
$$

Fix $g \in N$. From the above, it follows that

$$
a g b=c g^{\varphi} d .
$$

Now, by the first part of the lemma, and using (2) twice,

$$
\begin{aligned}
c\left(g^{\varphi}-g\right) d & =c g^{\varphi} d-(a b-c d) g-c g d \\
& =a[g, b]-c[g, d] \\
& \in \mathfrak{m}^{\operatorname{deg} a} \mathfrak{m}^{\operatorname{deg} b+p^{\epsilon}-1}+\mathfrak{m}^{\operatorname{deg} c} \mathfrak{m}^{\operatorname{deg} d+p^{\epsilon}-1} \\
& =\mathfrak{m}^{\operatorname{deg} c+\operatorname{deg} d+p^{\epsilon}-1} .
\end{aligned}
$$

Because $\operatorname{gr}_{\mathfrak{m}} \Omega_{N}$ is a domain [7, Theorem 7.24], we see that

$$
g^{\varphi}-g \in \mathfrak{m}^{p^{\epsilon}-1} \text {. }
$$

Hence by the second part of the lemma, we have

$$
g^{-1} g^{\varphi} \in\left(1+\mathfrak{m}^{p^{\epsilon}-1}\right) \cap N \leqslant N^{p^{\epsilon}}
$$

for all $g \in N$, so $[N, \varphi] \subseteq N^{p^{\epsilon}}$ as required.

2.2. Primeness - special cases. Let $G$ be a compact $p$-adic analytic group, and let $N$ be a closed normal uniform subgroup of $G$. Define

$$
E_{G}(N)=\left\{x \in G:[N, x] \subseteq N^{p^{\epsilon}}\right\} .
$$

This is the kernel of the conjugation action of $G$ on the finite set $N / N^{p^{\epsilon}}$ and as such is an open normal subgroup of $G$. Since $N$ is uniform, $N$ is always contained in $E_{G}(N)$.

We begin the proof of Theorem A by taking care of two special cases.

Proposition. Let $G$ be a compact p-adic analytic group. Suppose $N$ is an open normal uniform subgroup of $G$ such that $E_{G}(N)=N$. Then $\Omega_{G}$ is prime. 
Proof. We have observed in the Introduction that $\Omega_{G}$ is a crossed product of the Noetherian domain $\Omega_{N}$ with the finite group $\bar{G}:=G / N$. The set $S=\Omega_{N} \backslash\{0\}$ is a $\bar{G}$-invariant Ore set in $\Omega_{N}$, and $D_{N}=\Omega_{N} S^{-1}$ is the division ring of fractions of $\Omega_{N}$. By [15, Lemma 37.7], $S$ is an Ore set in $\Omega_{N} * \bar{G}$ consisting of regular elements, and

$$
\left(\Omega_{N} * \bar{G}\right) S^{-1} \cong D_{N} * \bar{G}
$$

Now, if $1 \neq \bar{g} \in \bar{G}$, conjugation by $\bar{g}$ gives an outer automorphism of $D_{N}$ by Proposition 2.1, because $E_{G}(N)=N$. By [12, Theorem 7.8.12], $D_{N} * \bar{G}$ is a simple ring. Since this is a partial quotient ring of $\Omega_{G}=\Omega_{N} * \bar{G}$, it follows that $\Omega_{G}$ is prime, as required.

We shall use below the elementary fact that, for any group $G$,

$$
\text { if } H \text { is a subgroup of } G \text { of finite index, then } \Delta^{+}(H) \leqslant \Delta^{+}(G) \text {. }
$$

Lemma. Let $G$ be a compact p-adic analytic group such that $\Delta^{+}(G)$ is a p-group. Let $N$ be a normal open uniform subgroup of $G$. Then $E_{G}(N)$ is a pro-p group.

Proof. Let $C=C_{G}(N)$ denote the centralizer of $N$ in $G$, so $C$ is closed by 21, Exercises 0.4(2)]. Let $q \neq p$ be a prime and let $Q$ be a $q$-Sylow subgroup of $C$; since $G$ is virtually pro-p, $Q$ is finite. Since $Q$ centralizes $N, Q$ is a finite normal subgroup of $N Q$ so $Q \leqslant \Delta^{+}(N Q) \leqslant \Delta^{+}(G)$ by (3). Hence $Q=1$ and $C$ is a pro-p group.

Now, consider the conjugation action of $E=E_{G}(N)$ on $N$. This has kernel $C \cap E$ which is a pro- $p$ group since $C$ is, and the image is contained in

$$
\Gamma:=\left\{\varphi \in \operatorname{Aut}(N):[N, \varphi] \subseteq N^{p^{\epsilon}}\right\} .
$$

By [7, Corollary 4.18], Aut $(N)$ may be identified with a subgroup of $G L_{d}\left(\mathbb{Z}_{p}\right)$ where $d=\operatorname{dim}(N)$. It is clear that under this identification $\Gamma$ is contained in $\left\{g \in G L_{d}\left(\mathbb{Z}_{p}\right): g \equiv 1\left(\bmod p^{\epsilon}\right)\right\}$ which is a pro-p group by [7, Theorem 5.2]. Hence $\Gamma$ is a pro $-p$ group and thus so is $E$ by [7, Proposition 1.11(ii)].

Corollary. Let $G$ be a compact p-adic analytic group such that $\Delta^{+}(G)=1$. Suppose that a $p$-Sylow subgroup $N$ of $G$ is normal and uniform. Then $E_{G}(N)=N$ and $\Omega_{G}$ is prime.

Proof. Since $E_{G}(N)$ contains $N$, the lemma shows that $E_{G}(N)=N$. Therefore $\Omega_{G}$ is prime by the proposition.

2.3. Proof of Theorem A. The proof needs a technical lemma which will be used to translate the group-theoretic condition $\Delta^{+}(G)=1$ into a condition involving crossed products. Compare the argument with the proof of [7, Lemma 2.2(iii)].

Lemma. Let $G$ be a pro-p group of finite rank such that $\Delta^{+}(G)=1$. Suppose $N$ is an open normal uniform subgroup of $G$ and let $x \in E=E_{G}(N)$. Then $H=\langle N, x\rangle$ is uniform.

Proof. Since $[H, H]=[N, H] \leqslant[N, E] \leqslant N^{p^{\epsilon}} \leqslant H^{p^{\epsilon}}, H$ is a finitely generated powerful pro-p group. By [7, Theorem 4.20], the elements of finite order in $H$ form a characteristic subgroup $T=\Delta^{+}(H)$ and $H / T$ is uniform. By (3), $T=1$ and the result follows.

Recall the definition of $w_{H, G}$ (or $w_{H}$ ) from (1.5). 
Proof of Theorem A. Suppose that $\Omega_{G}$ is prime. Let $e=\sum_{g \in \Delta^{+}(G)} g$, a nonzero central element of $\Omega_{G}$. Since $w_{\Delta^{+}(G)} . e \Omega_{G}=0, w_{\Delta^{+}(G)}=0$, and hence $\Delta^{+}(G)=1$.

Now suppose that $\Delta^{+}(G)=1$. Choose an open normal uniform subgroup $N$ of $G$, so that, by (1), $\Omega_{G}$ is a crossed product of the Noetherian domain $\Omega_{N}$ by the finite group $G / N$. Let $q$ be a prime (possibly equal to $p$ ) and let $H / N$ be a $q$-Sylow subgroup of $G / N$. By [14, Theorem 17.5], it is sufficient to show that $\Omega_{H}=\Omega_{N} *(H / N)$ is prime for all such $H$. If $q \neq p$, we see that $N$ is the $p$-Sylow subgroup of $H$; since $\Delta^{+}(H)=1$ by (3), $\Omega_{H}$ is prime by Corollary 2.2 ,

Suppose therefore that $q=p$, so $H / N$ is a finite $p$-group. By [14, Proposition 16.4] it is sufficient to prove that $\Omega_{H}=\Omega_{N} *(H / N)$ is semiprime. By [14, Theorem 18.10], this will follow from the semiprimeness of $\Omega_{P}$, where $P$ is an arbitrary subgroup of $H$ such that $N \subseteq P$ and $P / N$ is elementary abelian.

Let $P$ be any such group, and choose $K$, a maximal uniform subgroup of $P$ containing $N$. Since $P / N$ is abelian, $K$ is an open normal uniform subgroup of $P$. If $x \in E_{P}(K)$, then $\langle K, x\rangle$ is a uniform subgroup of $P$ containing $K$ by the lemma. The maximality of $K$ forces $x \in K$, so $E_{P}(K)=K$. Hence $\Omega_{P}$ is prime by Proposition 2.2, as required.

2.4. Proof of Theorem B. Choose an open normal uniform subgroup $N$ of $G$ contained in $P$. Then, as in (11), $\Omega_{G}=\Omega_{N} *(G / N)$ is a crossed product of the Noetherian domain $\Omega_{N}$ by the finite group $G / N$. It is clear that $P / N$ is a Sylow $p$-subgroup of $G / N$ and that $\Omega_{P}=\Omega_{N} *(P / N)$. The equivalence of (i) and (ii) now follows from [14, Corollary 18.11], whereas (ii) and (iii) are equivalent by [14, Proposition 16.4].

Now, (iii) and (iv) are equivalent by Theorem A. Since $P \cap \Delta^{+}(G)$ is a finite normal subgroup of $P, P \cap \Delta^{+}(G) \leqslant \Delta^{+}(P)$. Since $\Delta^{+}(P) \leq \Delta^{+}(G)$ by (3), $P \cap \Delta^{+}(G)=\Delta^{+}(P)$. But $P \cap \Delta^{+}(G)$ is a $p$-Sylow subgroup of $\Delta^{+}(G)$ by [21, Proposition 2.2.3(a)], and the equivalence of (iv) and (v) follows.

2.5. Proof of Theorem C. Suppose $G$ has torsion. Then we can find $1 \neq x \in G$ such that $x^{n}=1$. Now $(x-1)\left(x^{n-1}+\ldots+1\right)=0$ shows that $\Omega_{G}$ has zero divisors.

Now assume $G$ is torsion-free. The proof follows the line of Neumann's for $\Lambda_{G}$ 13] in appealing to a result of Walker [20] which guarantees that a semiprime Noetherian ring $R$ of finite global (homological) dimension, with $R / J(R)$ a field, is a domain. For certainly $\Omega_{G}$ is Noetherian, and it is semiprime by Theorem B. Any $q$-Sylow subgroup of $G$ with $q \neq p$ must be finite since $G$ is virtually pro $-p$. Hence $G$ is a pro- $p$ group and therefore $J\left(\Omega_{G}\right)=w_{G}$. Finally, the fact that $\Omega_{G}$ has finite global dimension follows from [4, Theorem 4.1] and [17, Corollaire 1].

\section{LOCALISABLE IDEALS IN $\Omega_{G}$}

3.1. Let $G$ be a compact $p$-adic analytic group and $H$ a closed normal subgroup of $G$. Recall the definition of $w_{H, G}$ from (1.5). When there is no danger of confusion, we will shorten $w_{H, G}$ to $w_{H}$. By [4, Lemma 4.5], $\Omega_{G}$ is a flat $\Omega_{H}$-module, so that

$$
w_{H, G}=w_{H, H} \Omega_{G}=(H-1) \Omega_{G}=\Omega_{G} w_{H, H} .
$$

Note that if $H$ is pro $-p$, then $w_{H, H}$ is the unique maximal ideal of $\Omega_{H}$.

Lemma. Let $H$ be a finite normal p-subgroup of $G$. Then $w_{H}$ is nilpotent. 
Proof. Since $H$ is a finite $p$-group, $\Omega_{H}=\mathbb{F}_{p}[H]$ is local Artinian with maximal ideal $\mathfrak{m}=w_{H, H}$, with $\mathfrak{m}^{n}=0$ for some $n$, by [15, Lemma 3.1.6]. Since $H$ is normal, $w_{H}^{n}=\left(\mathfrak{m} \Omega_{G}\right)^{n}=\mathfrak{m}^{n} \Omega_{G}=0$.

3.2. Now let $H$ be an arbitrary closed normal subgroup of $G$. By 7 , Corollary 8.34], $H$ contains an open pro- $p$ subgroup $J$, which can be chosen normal in $G$.

Let $P_{H} \triangleleft \Omega_{G}$ be the prime radical $\sqrt{w_{J}}$ of $w_{J}$, the smallest semiprime ideal of $\Omega_{G}$ containing $w_{J},[12,0.2 .8]$. By [12, Theorem 2.3.7], since $\Omega_{G}$ is Noetherian, $P_{H}$ is the biggest ideal of $\Omega_{G}$ which is nilpotent modulo $w_{J}$. The following lemma is essentially due to Peter Schneider, [6, Lemma 2.5].

Lemma. $P_{H}$ is independent of the choice of $J$.

Proof. Let $J$ and $K$ be open pro-p subgroups of $H$ which are normal in $G$. Then $J \cap K$ also has these properties, so it is sufficient to consider the case when $J \subseteq K$. Now $K / J$ is a finite normal $p$-subgroup of $G / J$, so $w_{K}^{n} \subseteq w_{J}$ for some $n$ by Lemma 3.1. Since $J \subseteq K, w_{J} \subseteq w_{K}$, so $\sqrt{w_{J}}=\sqrt{w_{K}}$.

It follows that if $H$ is itself pro- $p$, then $w_{H} \subseteq P_{H}$. If in addition $\Omega_{G / H}$ is semiprime, then $w_{H}=P_{H}$.

Our main result on localisable semiprime ideals is

Theorem D. $P_{H}$ is classically localisable in $\Omega_{G}$.

In particular, if $G$ is itself a pro- $p$ group and $H$ is a closed normal subgroup of $G$ with $\Delta^{+}(G / H)=1$, then $w_{H}$ is a prime localisable ideal of the local $\mathbb{F}_{p}$-algebra $\Omega_{G}$ by Theorems A and $\mathrm{D}$. One might imagine that in fact this is a special case of a general phenomenon, namely that every prime ideal $P$ of a local Noetherian $k$-algebra $R$ with $R / J(R) \cong k$ is classically localisable. But this is not so; for a counterexample see [5, Example 7.2], an example first studied by Ramras. However, in every such example known to us, there is a classically localisable semiprime ideal $Q$ contained in $P$, with $P$ a minimal prime ideal over $Q$; that is, in the jargon of [9], $P$ belongs to a finite clique. Indeed, the same is true in all known examples when $R$ is assumed to be merely semilocal rather than local.

3.3. Finite subgroups. We start the proof of Theorem D by taking care of a special case.

Proposition. Let $H$ be a finite normal subgroup of the compact p-adic analytic group $G$. Then $P_{H}$ is classically localisable in $\Omega_{G}$.

Proof. By Lemma 3.2, $P_{H}=\sqrt{w_{\{1\}}}=\sqrt{0}$ is the prime radical of $T=\Omega_{G}$. Pick an open normal uniform subgroup $N$ of $G$. As in the proof of Proposition 2.2, let $R=\Omega_{N}$ and $S=R \backslash\{0\}$; then $S$ is an Ore set in $T$ and

$$
T S^{-1} \cong\left(R S^{-1}\right) *(G / N)
$$

is a crossed product of a division ring with a finite group. Thus $T S^{-1}$ is Artinian, so any regular element in $T S^{-1}$ is a unit by [12, Proposition 3.1.1].

The natural map $T \rightarrow T S^{-1}$ is an injection, $T S^{-1}$ is a partial ring of quotients of $T$, and every regular element of $T$ is a unit in $T S^{-1}$. Hence $T S^{-1}$ is an Artinian (right and left) quotient ring of $T$, in the sense of 2.1.3 and 2.1.14 of [12]. Thus, the set $\mathcal{C}_{T}(0)$ of regular elements of $T$ must be an Ore set in $T$. By Small's Theorem [12, Corollary 4.1.4], $\mathcal{C}_{T}(0)=\mathcal{C}_{T}(\sqrt{0})$, so $\sqrt{0}$ is localisable in $T$ as required. Since 
$\sqrt{0} T S^{-1}$ is the nilpotent radical of $T S^{-1}$ it clearly has the Artin-Rees property, so $P_{H}$ is classically localisable.

3.4. The Artin-Rees property. The following result is inspired by Theorem 4.6 of $[18$.

Lemma. Let $N$ be a closed normal uniform subgroup of the compact p-adic analytic group $G$ and let $E=E_{G}(N)$. Then the graded ring of $\Omega_{G}$ with respect to the $w_{N^{-}}$ adic filtration is isomorphic to

$$
\Omega_{E / N}\left[X_{1}, \ldots, X_{d}\right] *(G / E),
$$

where $d=\operatorname{dim}(N)$, and is a Noetherian ring.

Proof. Let $\mathfrak{m}=w_{N, N}$ be the maximal ideal of $\Omega_{N}$, so that $w_{N}=\mathfrak{m} \Omega_{G}$. Since $w_{N}^{n}=\mathfrak{m}^{n} \Omega_{G}=w_{N, E}^{n} \Omega_{G}$, we see that

$$
\operatorname{gr}_{w_{N}} \Omega_{G} \cong\left(\mathrm{gr}_{w_{N, E}} \Omega_{E}\right) \otimes_{\Omega_{E}} \Omega_{G}
$$

as right $\Omega_{G}$-modules. Since $\operatorname{gr}_{w_{N, E}} \Omega_{E}$ is a subring and since $E$ has finite index in $G$, the ring $\operatorname{gr}_{w_{N}} \Omega_{G}$ is isomorphic to a crossed product of $\operatorname{gr}_{w_{N, E}} \Omega_{E}$ with $G / E$. We may hence assume that $E=G$, so $G$ acts trivially on $N / N^{p}$.

Next, $g N^{p} \mapsto g-1+\mathfrak{m}^{2}$ gives an isomorphism of the $G$-modules $N / N^{p}$ and $\mathfrak{m} / \mathfrak{m}^{2}$, so $G$ acts trivially on $\operatorname{gr}_{\mathfrak{m}} \Omega_{N}$. Now,

$$
\operatorname{gr}_{w_{N}} \Omega_{G} \cong\left(\operatorname{gr}_{\mathfrak{m}} \Omega_{N}\right) \otimes_{\Omega_{N}} \Omega_{G} \cong\left(\operatorname{gr}_{\mathfrak{m}} \Omega_{N}\right) \otimes_{\mathbb{F}_{p}} \Omega_{G / N} \cong \Omega_{G / N}\left[X_{1}, \ldots, X_{d}\right]
$$

as $\Omega_{G}$-modules, because $\operatorname{gr}_{\mathfrak{m}} \Omega_{N} \cong \mathbb{F}_{p}\left[X_{1}, \ldots, X_{d}\right]$ by Theorem 7.24 of [7]. Since $G$ acts trivially on $\operatorname{gr}_{\mathfrak{m}} \Omega_{N}$, this is a ring isomorphism.

Proposition. If $J$ is a closed normal uniform subgroup of $G$, then $w_{J} \triangleleft \Omega_{G}$ has the right and left Artin-Rees properties.

Proof. Let $N$ be an open normal subgroup of $G$. Since $N \cap J$ is open in $J$ it has ppower index in $J$. Thus $N J / N$ is a normal $p$-subgroup of $G / N$, so $w_{J}^{k} \subseteq w_{N J}^{k} \subseteq w_{N}$ for some $k \geqslant 0$, by Lemma 3.1. It follows that the $w_{J}$-adic topology is finer than the defining topology on $\Omega_{G}$ (where the base of open neighbourhoods of 0 is $\left.\left\{w_{N}: N \triangleleft_{o} G\right\}\right)$, so $\Omega_{G}$ is complete with respect to the $w_{J}$-adic filtration. Also, the graded ring of $\Omega_{G}$ with respect to the $w_{J}$-adic filtration is Noetherian by the lemma. By 8, Chapter II, Proposition 2.2.1], this filtration is Zariskian. Hence, by [8. Chapter II, Theorem 2.2], it has the Artin-Rees property, which is easily seen to imply that the ideal $w_{J}$ has the Artin-Rees property.

Since it is trivial to check that if an ideal $I$ of a Noetherian ring $R$ has the right Artin-Rees property, then so does $\sqrt{I}$, the following corollary is an immediate consequence of the proposition and the fact [7, Corollary 4.3] that we can find an open uniform subgroup of $H$ which is normal in $G$.

Corollary. $P_{H}$ has the Artin-Rees property whenever $H$ is a closed normal subgroup of $G$. 
3.5. Proof of Theorem D. Let $H$ be a closed normal subgroup of the compact $p$-adic analytic group $G$. Given Corollary 3.4, the theorem will follow if we show that $P_{H}$ is localisable. As before, let $J$ be an open uniform subgroup of $H$ which is normal in $G$. By Lemma 3.2, $P_{J}=P_{H}$, so we may assume that $H$ is actually uniform; in particular, $w_{H} \subseteq P_{H}=: P$. Since $P$ has the Artin-Rees property, [12, Theorem 4.2.10] shows that it is sufficient to prove that $P / P^{n}$ is localisable in $\Omega_{G} / P^{n}$ for all $n \geqslant 0$.

Now, the sets of ideals $\left\{w_{H, H}^{k}: k \geqslant 0\right\}$ and $\left\{w_{K, H}: K \triangleleft_{O} H\right\}$ are cofinal in $\Omega_{H}$ by [7, Lemma 7.1], so for all $n \geqslant 0$ there exists $K \triangleleft_{O} H$ such that $w_{K, H} \subseteq w_{H, H}^{n}$. By passing to an open subgroup, we may assume $K$ is normal in $G$. Therefore, $w_{K} \subseteq w_{H}^{n} \subseteq P^{n}$, so it is sufficient to show that $P_{H / K}=P / w_{K}$ is localisable in $\Omega_{G} / w_{K} \cong \Omega_{G / K}$. This follows directly from Proposition 3.3.

3.6. Centrally generated ideals. Both in the proof of Theorem E and when we come to consider the passage from localisability in $\Omega_{G}$ to localisability in $\Lambda_{G}$ in $₫ 4$ we need to lift Ore sets over a centrally generated ideal. In fact, for essentially no extra effort, we can lift over a polycentral ideal $I$ of the Noetherian ring $R$; that is, $I=\sum_{i=1}^{n} x_{i} R$, where $x_{1}$ is central in $R$, and, for $2 \leqslant j \leqslant n, x_{j}$ is central modulo $\sum_{i=1}^{j-1} x_{i} R$.

Proposition. Let $I$ be a polycentral ideal of the Noetherian ring $R$, with $I \subseteq P, P$ a semiprime ideal of $R$. Use $=$ to denote images in $\bar{R}:=R / I$. Then $P$ is classically (right) localisable in $R$ if and only if $\bar{P}$ is classically (right) localisable in $\bar{R}$.

Proof. One direction is trivial. So suppose for the non-trivial direction that $\bar{P}$ is classically right localisable in $\bar{R}$. By Noetherian induction we may assume that $I$ is generated by a single central element $x$ of $R$. We first show that $P / x^{n} R$ is localisable in $R / x^{n} R$ for all $n \geqslant 1$. By induction, assume that $n>1$ and that $P / x^{n-1} R$ is localisable in $R / x^{n-1} R$.

Let $r \in R$ and $c \in \mathcal{C}_{R}(P)$. We can find $r^{\prime} \in R$ and $c^{\prime} \in \mathcal{C}_{R}(P)$ such that $r c^{\prime}-c r^{\prime}=x^{n-1} u$ for some $u \in R$. We can also find $u^{\prime} \in R$ and $c^{\prime \prime} \in \mathcal{C}_{R}(P)$ such that $u c^{\prime \prime}-c u^{\prime}=x v$ for some $v \in R$. Hence, $r c^{\prime} c^{\prime \prime}-c r^{\prime} c^{\prime \prime}=x^{n-1} u c^{\prime \prime}=x^{n-1}\left(c u^{\prime}+x v\right)$, so $r\left(c^{\prime} c^{\prime \prime}\right)-c\left(r^{\prime} c^{\prime \prime}+x^{n-1} u^{\prime}\right)=x^{n} v$, as required.

Again, let $r \in R$ and $c \in \mathcal{C}_{R}(P)$. By [12, Proposition 4.2.6], $x R$ has the right Artin-Rees property, so there exists $n$ such that $(r R+c R) \cap(x R)^{n} \subseteq r x R+c x R$. By the above, we can find $r^{\prime} \in R$ and $c^{\prime} \in \mathcal{C}_{R}(P)$ such that $r c^{\prime}-c r^{\prime} \in x^{n} R$, so there exist $a, b \in R$ such that $r c^{\prime}-c r^{\prime}=r x a+c x b$. Hence $r\left(c^{\prime}-x a\right)=c\left(r^{\prime}+x b\right)$, and the result follows since $x \in P$.

Finally, the fact that the ideal $P R_{P}$ of $R_{P}$ has the Artin-Rees property follows from the facts that

- the image of $x$ in $R_{P}$ is central;

- $\overline{P R}_{\bar{P}}$ has the Artin-Rees property in $\bar{R}_{\bar{P}}$,

combined with the following statement: If an ideal $A$ of a Noetherian ring $S$ contains a polycentral ideal $B$, such that $A / B$ has the right Artin-Rees property in $S / B$, then $A$ has the right Artin-Rees property in $S$. This last statement can be proved by imitating the argument of [12, Theorem 4.2.7(i)].

The above proposition is a slight generalisation of a result of McConnell, [11, who dealt with the case $I=P$. See also [16, Theorem 2.2]. 
3.7. Proof of Theorem E. For this we need a group-theoretic result. Recall that a finite group is $p$-nilpotent if it has a normal subgroup of order prime to $p$, the factor by which is a $p$-group.

Proposition. Let $G$ be a compact p-adic analytic group such that $\Delta^{+}(G)$ is a $p$-group. Suppose that every factor of $G$ by an open normal subgroup is p-nilpotent. Then $G$ is a pro-p group.

Proof. Let $N$ be an open normal uniform subgroup of $G$. Then $N^{p^{\epsilon}}$ is also a normal subgroup of $G$, so $G / N^{p^{\epsilon}}$ is $p$-nilpotent. Hence we can find a normal $p^{\prime}$-subgroup $K / N^{p^{\epsilon}}$ of $G / N^{p^{\epsilon}}$ such that $G / K$ is a $p$-group. Since $N / N^{p^{\epsilon}}$ is a $p$-group, we see that $K \cap N \leqslant N^{p^{\epsilon}}$. It follows that $[K, N] \leqslant N^{p^{\epsilon}}$, so $K \leqslant E_{G}(N)$ which is a pro-p group by Lemma 2.2. Hence $K=N^{p^{\epsilon}}$, and therefore $G$ is a pro- $p$ group by [7, Proposition 1.11(ii)].

The following lemma is presumably well known, but we are unable to find a reference for it in the literature.

Lemma. Let $H$ be a finite normal subgroup of the compact p-adic analytic group $G$. Then $\Omega_{G}$ is a free $\Omega_{H}$-module.

Proof. Choose an open uniform normal subgroup $N$ of $G$. Since $N$ is torsion-free, $N \cap H=1$ so $H N \cong H \times N$. Now $\Omega_{H N}=\Omega_{H \times N} \cong \Omega_{H} \otimes_{\mathbb{F}_{p}} \Omega_{N}$ because $H$ is finite, so $\Omega_{H N}$ is a free $\Omega_{H}$-module. Since $\Omega_{G}$ is a free $\Omega_{H N}-$ module, our result follows.

Proof of Theorem E. Let $F=\Delta_{p^{\prime}}^{+}(H)$. Since $p \nmid|F|$, it is well known that $w_{F, F}$ is generated by the central idempotent

$$
f=1-\frac{1}{|F|} \sum_{g \in F} g
$$

so $w_{F}=f \Omega_{G}$ and $\Omega_{G} / f \Omega_{G} \cong \Omega_{G / F}$. Since $F$ is normal in $G, f$ is central in $\Omega_{G}$.

(iii) $\Rightarrow$ (ii): By Proposition 3.6 we may assume that $F=1$, so $H$ is pro- $p$. Since $\Omega_{G / H}$ is semiprime, $w_{H, G}=P_{H}$, and the result follows from Theorem D.

(ii) $\Rightarrow$ (i): Trivial.

(i) $\Rightarrow$ (iii): Suppose that $w_{H, G}$ is localisable. Without loss of generality we can assume that $F=1$.

Let $V$ be any open normal subgroup of $H$. We claim that $H / V$ is $p$-nilpotent. Since quotients of finite $p$-nilpotent groups are $p$-nilpotent, we may assume that $V$ is normal in $G$ by passing to an open subgroup. Writing $=$ for images in $G / V$, $w_{\bar{H}, \bar{G}}$ is localisable in $\Omega_{\bar{G}}$.

Since $\Omega_{\bar{G}}$ is a free $\Omega_{\bar{H}}$-module by the lemma, it is easy to deduce that $w_{\bar{H}}$ is a localisable maximal ideal of $\Omega_{\bar{H}}$. That is, the augmentation ideal of the group algebra $\mathbb{F}_{p} \bar{H}$ is localisable. Therefore, by [15, Theorem 11.2.15], $\bar{H}$ is $p$-nilpotent, as claimed.

In particular, $\Delta^{+}(H)$ is $p$-nilpotent. Since $F=1, \Delta^{+}(H)$ is a $p$-group. Therefore the proposition shows that $H$ is pro- $p$, completing the proof of the theorem. 


\section{Lifting information to $\Lambda_{G}$}

4.1. Let $G$ be a compact $p$-adic analytic group. As was noted in (1.2), $\Lambda_{G}$ is semilocal with $p$ a central regular element in $J\left(\Lambda_{G}\right)$, such that

$$
\Lambda_{G} / p \Lambda_{G} \cong \Omega_{G} \text {. }
$$

These properties establish a strong connection between $\Lambda_{G}$ and $\Omega_{G}$, and we use them to deduce corresponding results for $\Lambda_{G}$ that we established for $\Omega_{G}$ in the previous sections.

4.2. Primeness, semiprimeness and zero divisors. The first and last parts of the following result are simply stated for completeness and are due to Neumann, 13. The proof of (i) was noted in (1.3); (iii) can if desired be deduced from Theorem $\mathrm{C}$ by an easy lifting argument. Similarly, it is easy to deduce (ii) from Theorem A, noting that $\bigcap_{i \geqslant 0} p^{i} \Lambda_{G}=0$.

Theorem F. Let $G$ be a compact p-adic analytic group. Then

(i) $\Lambda_{G}$ is semiprime;

(ii) $\Lambda_{G}$ is prime if and only if $\Delta^{+}(G)=1$;

(iii) $\Lambda_{G}$ is a domain if and only if $G$ is torsion-free.

4.3. Localisation in $\Lambda_{G}$. As an immediate consequence of Proposition 3.6 and Theorem $\mathrm{D}$, we can state

Theorem G. Let $G$ be a compact p-adic analytic group and let $H$ be a closed normal subgroup. Let $I_{H}$ be the inverse image of the ideal $P_{H}$ of $\Omega_{G}$ in $\Lambda_{G}$. Then $I_{H}$ is classically localisable in $\Lambda_{G}$.

In view of [6. Proposition 2.6], this is a direct generalisation of [6, Theorem 2.4] from the case when $G / H \cong \mathbb{Z}_{p}$ to the case of an arbitrary closed normal subgroup $H$ of $G$.

Theorem H. Let $G$ be a compact p-adic analytic group and let $H$ be a closed normal subgroup of $G$ such that $\Omega_{G / H}$ is semiprime. Let $v_{H, G}$ denote the inverse image of $w_{H, G}$ in $\Lambda_{G}$. Then the following are equivalent:

(i) $v_{H, G}$ is localisable;

(ii) $v_{H, G}$ is classically localisable;

(iii) $H / \Delta_{p^{\prime}}^{+}(H)$ is pro-p.

Proof. Let us write $(i)_{\Lambda},(i i)_{\Lambda},(i)_{\Omega},(i i)_{\Omega}$ for the relevant statements in Theorems $\mathrm{H}$ and E, respectively. Using Proposition 3.6 and Theorem E it is easy to obtain the chain of implications

$$
(i i)_{\Lambda} \Rightarrow(i)_{\Lambda} \Rightarrow(i)_{\Omega} \Leftrightarrow(i i i) \Leftrightarrow(i i)_{\Omega} \Leftrightarrow(i i)_{\Lambda}
$$

proving the result.

\section{Krull AND GLOBAl Dimensions}

5.1. Let $G$ be a compact $p$-adic analytic group, and let $H$ be a closed normal subgroup. By Theorem $\mathrm{D}$, the semiprime ideal $P_{H}=P_{H, G}$ is always localisable in $\Omega_{G}$. We will denote the localisation of $\Omega_{G}$ at $P_{H, G}$ by $\Omega_{G, H} . \Lambda_{G, H}$ is defined analogously.

Now suppose that $K$ is an open uniform subgroup of $G$. Let $T /(H \cap K)=$ $\Delta^{+}(K /(H \cap K))$; since $K /(H \cap K)$ is powerful, $K / T$ is uniform by [7, Theorem 
4.20]. Since $H \cap K$ is open in both $T$ and $H, \Omega_{G, H}=\Omega_{G, T}$ by Lemma 3.2 so we may assume that $H=T$. It follows from [7, Proposition 4.31(ii)] that $H$ is uniform.

That is, we will assume for the remainder of this section that $G$ is a compact $p$-adic analytic group containing an open normal uniform subgroup $K$ and a closed normal subgroup $H$ such that $H \subseteq K$, and both $H$ and $K / H$ are uniform. Note that these conditions imply $[H, K] \subseteq H \cap[K, K] \subseteq H^{p^{\epsilon}}$, whence $E_{K}(H)=K$.

Lemma. $\Omega_{G, H}$ is a crossed product of $\Omega_{K, H}$ with the finite group $G / K$ :

$$
\Omega_{G, H}=\Omega_{K, H} *(G / K) .
$$

There is an analogous statement for $\Lambda_{G, H}$.

Proof. We have observed earlier that $\Omega_{G}=\Omega_{K} *(G / K)$. Let $S=\mathcal{C}_{\Omega_{K}}\left(P_{H, K}\right)$, an Ore set in $\Omega_{K}$ by Theorem D. Since $K, H$ and $K / H$ are uniform, $P_{H, K}=w_{H, K}$, and $S=\Omega_{G} \backslash w_{H, K}$ consists of regular elements of $\Omega_{K}$. Since $H$ and $K$ are normal in $G, S$ is $G$-invariant and is hence an Ore set in $\Omega_{G}$ consisting of regular elements by [14, Lemma 37.7]. Moreover,

$$
\Omega_{G} S^{-1}=\Omega_{K} S^{-1} *(G / K)=\Omega_{K, H} *(G / K) .
$$

Now, as in the proof of Proposition [3.3. $\Omega_{G / H}$ has an Artinian quotient ring, so $\mathcal{C}_{\Omega_{G / H}}(0)=\mathcal{C}_{\Omega_{G / H}}(\sqrt{0})$ by [12, Corollary 4.1.4]. Hence $T:=\mathcal{C}_{\Omega_{G}}\left(P_{H, G}\right)=$ $\mathcal{C}_{\Omega_{G}}\left(w_{H, G}\right)$. It is easy to check that $S \subseteq T$, so we have a ring homomorphism

$$
\Omega_{K, H} *(G / K)=\Omega_{G} S^{-1} \longrightarrow \Omega_{G} T^{-1}=\Omega_{G, H} .
$$

To show that this map is an isomorphism, it is sufficient to show that $T \subseteq \Omega_{G} \subseteq$ $\Omega_{G} S^{-1}$ consists of units in $\Omega_{G} S^{-1}$. To this end, observe that

$$
\Omega_{G} S^{-1} / w_{H, G} S^{-1} \cong\left(\Omega_{K / H} *(G / K)\right) \bar{S}^{-1} \cong D_{K / H} *(G / K)
$$

is an Artinian ring, where $D_{K / H}$ is the division ring of fractions of $\Omega_{K / H}$ and $\bar{S}=\Omega_{K / H} \backslash\{0\}$. Using [12, Proposition 2.1.16(iv)] it is easy to show that any element $x \in \Omega_{G}$ which is regular modulo $w_{H, G}$ is regular modulo $w_{H, G} S^{-1}$ inside $\Omega_{G} S^{-1}$. Since any regular element in an Artinian ring is a unit 12, Proposition 3.1.1], we can find $y \in \Omega_{G} S^{-1}$ such that $x y \equiv 1 \bmod w_{H, G} S^{-1}$. Now, $w_{H, K} S^{-1}$ is the Jacobson radical of $\Omega_{K, H}$, so $w_{H, K} S^{-1}$ is contained in the Jacobson radical $J$ of $\Omega_{G} S^{-1}=\Omega_{K, H} *(G / K)$ by [14, Theorem 4.2]. Hence $w_{H, G} S^{-1} \subseteq J$, so $x y \equiv 1$ mod $J$. It follows that $x$ is a unit in $\Omega_{G} S^{-1}$, as required.

The argument to handle $\Lambda_{G, H}$ is similar.

5.2. The following result will be very useful in the computation of Krull and global dimensions of $\Omega_{G, H}$.

Lemma. $\Omega_{G, H}$ is a faithfully flat $\Omega_{H}-$ module.

Proof. By [4, Lemma 4.5] $\Omega_{G}$ is a flat $\Omega_{H}$-module. Because localisation is flat, it follows that $\Omega_{G, H}$ is a flat $\Omega_{H}$-module. Since $\Omega_{H}$ has a unique maximal right (and left) ideal $\mathfrak{m}=w_{H, H}$, to complete the proof it is sufficient to prove that $\mathfrak{m} \Omega_{G, H} \neq \Omega_{G, H}$ by [12, Proposition 7.2.3]. But $\mathfrak{m} \Omega_{G, H}$ is contained in the Jacobson radical of $\Omega_{G, H}$ and is hence a proper ideal of $\Omega_{G, H}$, as required. 
5.3. Krull dimension. To obtain the upper bounds in Theorem I we require

Proposition. Let $J=w_{H, K} \Omega_{K, H}$ denote the unique maximal ideal of $\Omega_{K, H}$. Then the $J$-adic filtration on $\Omega_{K, H}$ is Zariskian and the graded ring of $\Omega_{K, H}$ with respect to this filtration is isomorphic to

$$
D_{K / H}\left[X_{1}, \ldots, X_{d}\right]
$$

where $D_{K / H}$ denotes the division ring of fractions of $\Omega_{K / H}$ and $d=\operatorname{dim} H$.

Proof. Write $I$ for $w_{H, K}$. We have observed in the proof of Proposition 3.4 that the $I$-adic filtration on $\Omega_{K}$ is Zariskian. Hence the Rees ring

$$
\widetilde{\Omega_{K}}=\cdots \oplus I^{2} t^{-2} \oplus I t^{-1} \oplus \Omega_{K}[t]
$$

is Noetherian. Since $S=\Omega_{K} \backslash I$ is an Ore set in $\Omega_{K}$, it is easily checked that $S \subseteq \widetilde{\Omega_{K}}$ is an Ore set in $\widetilde{\Omega_{K}}$. Hence

$$
\widetilde{\Omega_{K}} S^{-1}=\cdots \oplus J^{2} t^{-2} \oplus J t^{-1} \oplus \Omega_{K, H}[t] \cong \widetilde{\Omega_{K, H}}
$$

is Noetherian. Since $J$ is the Jacobson radical of $\Omega_{K, H}$, the $J$-adic filtration is Zariskian.

The second assertion follows easily from Lemma 3.4, since $E_{K}(H)=K$.

Proof of Theorem I. This is now just a matter of putting all the pieces together. By Lemma 5.2 and [12, Lemma 6.5.3(i)], $\mathcal{K}\left(\Omega_{H}\right) \leqslant \mathcal{K}\left(\Omega_{G, H}\right)$. Now $\mathcal{K}\left(\Omega_{G, H}\right)=$ $\mathcal{K}\left(\Omega_{K, H}\right)$ by Lemma [5.1 and [12, Proposition 10.1.11(ii)].

Since $\mathcal{K}\left(D_{K / H}\left[X_{1}, \ldots, X_{d}\right]\right)=d$ by [12, Proposition 6.5.4(i)], part (i) follows from the Proposition and 8, Chapter II, Corollary 3.1.3].

Now, $\mathcal{K}\left(\Lambda_{G, H}\right) \leqslant \mathcal{K}\left(\Lambda_{G}\right)$, and this is finite by [1, Theorem A]; moreover, the element $p$ of $I_{H} \subseteq J\left(\Lambda_{G, H}\right)$ is a central regular element of $\Lambda_{G, H}$. Since $\Lambda_{G, H} / p \Lambda_{G, H} \cong$ $\Omega_{G, H}$, the second part follows from [20. Theorem 1.8]. (See also [1, Theorem C].)

5.4. Homological properties. Before embarking on the proofs, we recall that a Noetherian ring $R$ is Auslander-Gorenstein if it has finite right and left injective dimensions, $n$ say, and the Auslander property holds for all finitely generated right and left $R$-modules $M$ : that is, for all integers $i, 0 \leqslant i \leqslant n$, and for all $j<i$ and all submodules $N$ of $\operatorname{Ext}_{R}^{i}(M, R)$, $\operatorname{Ext}_{R}^{j}(N, R)=0$. An Auslander-regular ring is one which is Auslander-Gorenstein and has finite global dimension.

The following lemma is surely well known, but we have been unable to locate it in the literature. Note that it is actually concerned with Frobenius extensions, as defined, for instance, in [3. The proof of the lemma, coupled with the simple application of it outlined in the proof of Theorem J, shows that a Frobenius extension of an Auslander-Gorenstein ring is Auslander-Gorenstein.

Lemma. Let $T=R * G$ be a crossed product of the ring $R$ with the finite group $G$ and let $M_{T}$ be a right $T$-module. Then

$$
\operatorname{Ext}_{T}^{i}(M, T) \cong \operatorname{Ext}_{R}^{i}(M, R)
$$

as left $R$-modules, for all $n \geqslant 0$. 
Proof. As functors from the category of right $T$-modules to the category of left $R$-modules, $\operatorname{Ext}_{R}^{i}(-, R)$ and $\operatorname{Ext}_{T}^{i}(-, T)$ are derived from $\operatorname{Hom}_{R}\left(-, R_{R}\right)$ and from $\operatorname{Hom}_{T}\left(-, T_{T}\right)$ respectively. It is therefore sufficient to prove that

$$
\operatorname{Hom}_{T}\left(M_{T}, T_{T}\right) \cong \operatorname{Hom}_{R}\left(M_{R}, R_{R}\right)
$$

as left $R$-modules. Since $M_{R} \cong M_{T} \otimes_{T} T_{R}$, it is enough to show that $T \cong$ $\operatorname{Hom}_{R}\left(T_{R}, R_{R}\right)$ as right $T$-modules.

Define $\alpha: \operatorname{Hom}_{R}\left(T_{R}, R_{R}\right) \rightarrow T$ by $\alpha(f)=\sum_{g \in G} f(\bar{g}) \bar{g}^{-1}$. An easy check shows that $\alpha$ is a right $T$-module map. Since $\beta: T \rightarrow \operatorname{Hom}_{R}\left(T_{R}, R_{R}\right)$ given by $\beta\left(\sum_{g \in G} r_{g} \bar{g}\right)(\bar{h})=r_{h^{-1}}$ is an inverse, the result follows.

Corollary. Let $R=T * G$ be the crossed product of a ring $R$ with a finite group $G$. Then the injective dimensions of $R$ and $T$ viewed as right modules over $R$ and $T$, respectively, are equal.

Proof of Theorem J. We first prove the claims concerning global dimension.

(iii) Choose an open normal uniform subgroup $K$ of $G$ and continue with the reductions made in Section 5.1, so in particular $H \subseteq K \subseteq G$.

Suppose that $G$ contains no elements of order $p$. By [4, Theorem 4.1] and 17. Corollaire 1], the global dimension of $\Omega_{G}$ and hence of $\Omega_{G, H}$ is finite. By Lemma 5.1, $\Omega_{G, H}$ is the crossed product of $\Omega_{K, H}$ with the finite group $G / K$, so $\operatorname{gld}\left(\Omega_{G, H}\right)=\operatorname{gld}\left(\Omega_{K, H}\right)$ by [22, Lemma $\left.2.2(\mathrm{i})\right]$.

By Lemma 5.2, [12, Theorem 7.2.6] and the results of Brumer and Serre [4, 17,

$$
d:=\operatorname{dim} H=\operatorname{gld}\left(\Omega_{H}\right) \leqslant \operatorname{gld}\left(\Omega_{K, H}\right) .
$$

By Proposition 5.3 we know that $\Omega_{K, H}$ has a Zariskian filtration with graded ring $D_{K / H}\left[X_{1}, \ldots, X_{d}\right]$, so we may deduce from [12, Theorem 7.5.3(iii)] and [8, Chapter II, Theorem 3.1.4] that

$$
\operatorname{gld}\left(\Omega_{K, H}\right) \leqslant \operatorname{gld}\left(D_{K / H}\left[X_{1}, \ldots, X_{d}\right]\right)=d
$$

and the first set of displayed values in (iii) follows. The second display follows easily from [12, Theorem 7.3.7], since $\Lambda_{G, H} / p \Lambda_{G, H} \cong \Omega_{G, H}$.

(iv) Suppose that $1 \neq x \in G$ with $x^{p}=1$ and $x H \in \Delta^{+}(G / H)$, and suppose for a contradiction that gld $\Lambda_{G, H}<\infty$. Let $C=\{g \in G:[g, x] \in H\}$, the centralizer in $G$ of $x H$; this is an open subgroup of $G$. Choose a uniform subgroup $K$ of $C$ which is open and normal in $G$. By changing $H$ if necessary as in Section [5.1] we can assume that $H \leqslant K$ and that both $H$ and $K / H$ are uniform. Moreover, we still have the property that $[K, x] \subseteq H$.

By Lemma 5.1, we have

$$
\Lambda_{G, H} \cong \Lambda_{K, H} *(G / K) .
$$

Set $E=\langle K, x\rangle=K \rtimes\langle x\rangle$, so $\Lambda_{E, H}=\Lambda_{K, H} *\langle x\rangle$ is a skew group ring. By 22, Lemma 2.1(ii)] and the above isomorphism, gld $\Lambda_{K, H} *\langle x\rangle<\infty$.

Write $J$ for the Jacobson radical of $\Lambda_{K, H}$. Since $K / H$ is uniform, $\Lambda_{K, H} / J$ is isomorphic to the division ring of fractions $F$ of $\Omega_{K / H}$. Thus [22, Theorem 4.5(ii)] implies that $F *\langle x\rangle$ is semisimple Artinian. But $[K, x] \subseteq H$ and $F$ has characteristic $p$, so $x-1$ is a non-zero central nilpotent element of $F *\langle x\rangle$. This contradiction shows that gld $\Lambda_{G, H}$ must be infinite.

Finally, if gld $\Omega_{G, H}<\infty$, then gld $\Lambda_{G, H}<\infty$ by [12, Theorem 7.3.7], so this case follows as well. 
(i) Lemma 5.1 and the Lemma proved above show that it is enough to prove the result for $\Omega_{K, H}$. For the latter algebra, the Auslander-Gorenstein property is a consequence of Proposition [5.3 and [8, Chapter III, Theorem 2.2.5], since a polynomial ring over a division ring is Auslander regular by [8, Chapter III, Theorem 2.3.5]. The value of the injective dimension follows from the global dimension calculation and the corollary above.

(ii) Filter $\Lambda_{G, H}$ using the powers of the ideal $p \Lambda_{G, H}$. The associated graded algebra is isomorphic to $\Omega_{G, H}[X]$, and hence is Auslander-Gorenstein by (i) and [8. Chapter III, Theorem 2.3.5]. Since the filtration is separated and complete, we deduce that $\Lambda_{G, H}$ is also Auslander-Gorenstein using [8, Chapter III, Theorem 2.2.5]. The value of the injective dimension follows as in (i).

Whilst the condition that $G$ has no elements of order $p$ is certainly sufficient in order for $\Omega_{G, H}$ and $\Lambda_{G, H}$ to have finite global dimension, it is not necessary. To see this, take any compact $p$-adic analytic group $G$ with elements of order $p$ but such that $\Delta^{+}(G)=1$ (the wreath product of $\mathbb{F}_{p}$ with $\mathbb{Z}_{p}$ is an example). Then $\Omega_{G}$ is prime by Theorem A. Letting $H=1$, we see that $\Omega_{G, H}$ is the simple Artinian ring of quotients of $\Omega_{G}$ and as such has global dimension 0 . We believe that in fact the necessary condition of Theorem J(iv) is also sufficient.

Conjecture. The global dimension of $\Omega_{G, H}$ (and of $\Lambda_{G, H}$ ) is infinite if and only if there exists an element $x \in G$ of order $p$ such that the centralizer of $x H$ in $G$ is open in $G$.

\section{NOTE ADDED IN PROOF}

This conjecture has now been proved by the first author, [2].

\section{REFERENCES}

1. K. Ardakov, Krull dimension of Iwasawa Algebras, J. Algebra 280 (2004), 190-206. MR2081928 (2005e:16036)

2. K. Ardakov, Localisation at augmentation ideals in Iwasawa algebras, Glasgow Math J. 48 (2006), 251-267.

3. A. Bell and R. Farnsteiner, On the theory of Frobenius extensions and its application to Lie superalgebras, Trans. Amer. Math. Soc. 335 (1993), 407-424. MR.1097163 (93c:17049)

4. A. Brumer, Pseudocompact algebras, profinite groups and class formations, J. Algebra 4 (1966), 442-470. MR0202790 (34:2650)

5. K.A. Brown, C.R. Hajarnavis and A.E. MacEacharn, Rings of finite global dimension integral over their centres, Comm. in Algebra 11 (1983), 67-93. MR0687406 (84b:16029)

6. J. Coates, T. Fukaya, K. Kato, R. Sujatha, O. Venjakob, The $G L_{2}$ main conjecture for elliptic curves without complex multiplication, preprint, arXiv math NT/0404297.

7. J.D. Dixon, M.P.F. Du Sautoy, A. Mann, D. Segal, Analytic pro-p groups, 2nd edition, CUP (1999). MR 1720368(2000m:20039)

8. L. Huishi and F. van Oystaeyen, Zariskian filtrations, Kluwer Academic Publishers, Kmonographs in Mathematics, vol. 2 (1996). MR1420862 (97m:16083)

9. A. V. Jategaonkar, Localization in Noetherian Rings, London Math. Soc. Lecture Notes vol. 98, CUP, 1986. MR0839644 (88c:16005)

10. M. Lazard, Groupes analytiques p-adiques, Publ. Math. IHES 26 (1965), 389-603. MR0209286 $(35: 188)$

11. J.C. McConnell, Localisation in enveloping rings, J. London Math. Soc. 43 (1968), 421-428; Erratum and Addendum, ibid. (2) 3, 409-410. MR0228532(37:4112) MR0313341 (47:1896)

12. J.C. McConnell, J.C. Robson, Noncommutative Noetherian rings, AMS Graduate Studies in Mathematics, vol. 30. MR.1811901 (2001i:16039) 
13. A. Neumann, Completed group algebras without zero-divisors, Arch. Math. Basel 51 (1988), 496-499. MR0973723 (90a:20014)

14. D. Passman, Infinite Crossed Products, Pure and Applied Mathematics vol. 135, Academic Press, San Diego (1989). MR0979094 (90g:16002)

15. D. Passman, The Algebraic Structure of Group Rings, Wiley-Interscience, New York (1977). MR0470211 (81d:16001)

16. P. F. Smith, Localisation and the AR property, Proc. Lond. Math. Soc. 2 (1971), 39-68. MR0294383 (45:3453)

17. J.-P. Serre, Sur la dimension homologique des groupes profinis, Topology 3 (1965), 413-420. MR 0180619 (31:4853)

18. O. Venjakob, Characteristic elements in non-commutative Iwasawa theory, J. Reine Angew. Math. 583 (2005), 193-236. MR2146857 (2006d:11133)

19. O. Venjakob, On the structure theory of the Iwasawa algebra of a compact p-adic Lie group, J. Eur. Math. Soc. (JEMS) 4 (2002), no. 3, 271-311. MR.1924402 (2004h:16029)

20. R. Walker, Local rings and normalizing sets of elements, Proc. Lond. Math. Soc. 24 (1972), 27-45. MR0294399 (45:3469)

21. J. S. Wilson, Profinite Groups, Lond. Math. Soc. Monographs 19, Oxford University Press, Oxford, 1998. MR.1691054 (2000j:20048)

22. Y. Zhong, Homological dimension of crossed products, J. Algebra 164 (1994), 101-123. MR 1268329 (95a:16039)

Department of Pure Mathematics and Mathematical Statistics, University of CamBridge, Cambridge CB3 0WB, United Kingdom

E-mail address: K.Ardakov@dpmms.cam.ac.uk

Department of Mathematics, University of Glasgow, Glasgow G12 8QW, United KINGDOM

E-mail address: kab@maths.gla.ac.uk 